\title{
State-of-the-Art Digital Compact Pyrometer Series
}

\author{
D. Wagner, G. Hofmann, DIAS Infrared GmbH; \\ R. Breternitz, C. Tzschaschel, B. Dohmeyer, D. Franke, M. Kerl, DIAS Systems GmbH \\ Gostritzer Str. 63, 01217 Dresden, Germany \\ www.dias-infrared.com
}

\section{Introduction}

State-of-the-art stationary digital pyrometers for non-contact temperature measurement are evolving in enhanced features and characteristics.

This development takes place even with reducing prices for these products.

Large varieties for easy adjustment to variable measuring tasks combined with efficient digital signal processing characterize today's pyrometers.

In the following some basic knowledge and device type examples about new pyrometer series

PYROSPOT from DIAS Infrared $\mathrm{GmbH}$ will be introduced.

\section{General design and functional principles}

Many known pyrometers are equipped with infrared detectors based on Silicon or Germanium or Indium Gallium Arsenide photodiodes. Well known are also devices with thermopile or pyroelectric detectors. Other detectors based on photoconductive materials e.g. PbS and PbSe are also very common.

The infrared radiation from the measuring object is transferred through the optical path to the detector. The detector generates an electrical signal which can be further processed by electronics and micro processors.

There are several types of optics possibilities

- Optics with fixed sharp point at given distance

- Optics with focussable sharp point

- Fibre optics cable with optical heads used for high ambient temperatures and measuring tasks where the object can be hardly reached

These optics can be equipped with protection windows from different materials due to spectral range to avoid damage and pollution of the lens.

The alignment of the pyrometer to the measuring object is realized with LED or LASER aiming lights, through lens sighting or video output. 
The signal processing is divided into analogue and digital part.

If for example a photodiode is used as infrared detector, this photodiode generates a current which is proportional to the infrared radiation. This current signal can be reaching over six decades at common measuring ranges. The emissivity correction adds two more decades.

This signal has to be preprocessed with the aim to compress dynamics range and prepare adaption to $A / D$ converter. The $A / D$ converter and following micro processor calculate a temperature signal.

The micro processor (e.g. 8051 with high performance core) has the task of:

- $\mathrm{A} / \mathrm{D}$ conversion of compressed photodiode current

- Temperature compensation with determination of internal sensor temperature

- Emissivity correction

- Calculation of temperature and linear output signal, e.g. 4...20 mA

- Realization of digital interface for data transfer or device settings, e.g. RS 485 or USB interfaces

Depending on device features other functions may be also necessary, such as:

- Display and operating keys directly on the device

- Controlling of integrated video camera including chroma keying of important parameters into the picture

- Special processing algorithms for certain applications

The digital pyrometer protocol has been realized on MODBUS-RTU /1//2/ basis.

MODBUS-RTU is worldwide common protocol structure for master-slave communication.

Typical MODBUS request structure contains:

- Slave address

- Command code (e.g. read or write register)

- Data

- $\quad$ CRC ckeck sum

Typical MODBUS answer structure contains:

- Slave address

- Command code as echo from request

- desired data (read) or echo data from request (write)

- $\quad$ CRC ckeck sum

The MODBUS protocol is independent of physical layer (RS 485, RS 232 or USB).

The baud rate within the pyrometer can be up to $115 \mathrm{kBd}$ mostly via the RS 485 interface with half duplex mode. The common register size is 16 Bit.

Parameter dimensions have been defined to use this data size optimally:

- Temperatures: in kelvin * 16

- Emissivitiy as \%-value * 10

- Discrete time steps, e.g. response times in logarithmic steps 
Some examples from the comprehensive table of defined registers are shown in Table 1.

Table 1: Examples for defined registers in MODBUS protocol

\begin{tabular}{|l|l|l|l|r|}
\hline $\begin{array}{l}\text { Register- } \\
\text { address }\end{array}$ & R/W & Description & \multicolumn{2}{|c|}{ Data 16 Bit } \\
\cline { 4 - 5 } & & MSB & LSB \\
\hline 0100 & $\mathrm{R}$ & Measurement status & Status & Time Stamp \\
\hline 0101 & $\mathrm{R}$ & Measuring temperature & Byte1 & Byte0 \\
\hline 0102 & $\mathrm{R} / \mathrm{W}$ & Emissivity & Byte1 & Byte0 \\
\hline 0103 & $\mathrm{R} / \mathrm{W}$ & Response time & 0 & Time \\
\hline 0104 & $\mathrm{R} / \mathrm{W}$ & Clear mode / clear time & Mode & Time \\
\hline
\end{tabular}

All registers can be read or write separate or as block of max. 8 registers. Write registers offers possibility to read out parameter limits.

Between the CRC check sum also error handling is done according to MODBUS specifications within the transfer layer.

\section{Pyrometer type examples}

The digital Pyrometers of PYROSPOT series 40 and 44 (Fig. 1 and 2) are especially designed for industrial purpose. The devices are suitable for temperature measurement on many different metallic or non-metallic surfaces.

The special spectral range of DT $40 G$ and DT $44 G$ makes the device suitable for measurement of glass surfaces. The DT 40F and DT 44F is suitable for measurement through flames and combustions gases by working in also special spectral range.

The digital pyrometers PYROSPOT series 10 (Fig.3 and 4) are also designed for industry and research applications. The devices are suitable for temperature measurement from $200{ }^{\circ} \mathrm{C}$ of many different surfaces.

Several Fixed-, Vario- or Fibre optics realizes small measuring spot sizes.

The temperature linear standard output signal of 0/4 to $20 \mathrm{~mA}$ allows easy implementation in existing measuring and controlling systems.

The solid body in compact housing allows usage even under rough environmental conditions. With fast response times these Pyrometers are also suitable for fast measuring processes.

The PYROSPOT series possesses several types of digital interfaces.

The devices of series 40 are equipped with integrated, galvanically isolated USB interface, which allows parameterizing and software evaluation even without connecting extra power supply.

Series 44 and series 10 are equipped with galvanically isolated RS 485 interface for BUS system integration and modules for USB connection.

Integrated LED or laser aiming light or, alternative, the true sided through-lens sighting, enables to focus the measuring object exactly.

All parameters can be easily adjusted to the application by using the comfortable parameterizing and evaluation software PYROSOFT Spot.

Tables 2, 3 and 4 show an overview about technical data of PYROSPOT series 40, 44 and 10. 


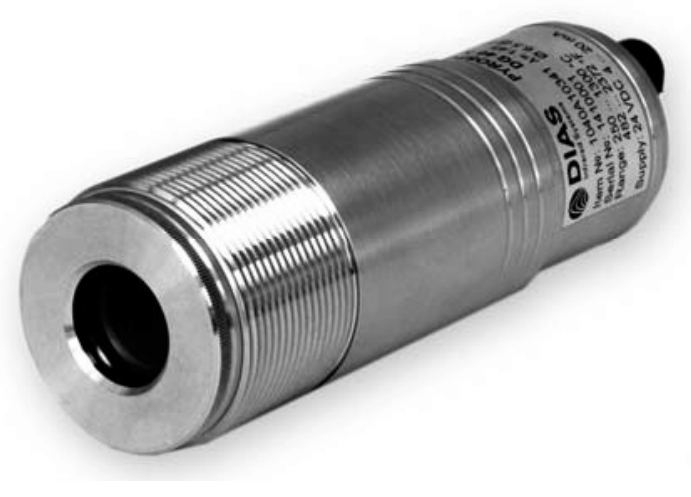

Fig.1: PYROSPOT Series $\mathbf{4 0}$ and $\mathbf{4 4}$ with fixed or focussable optics

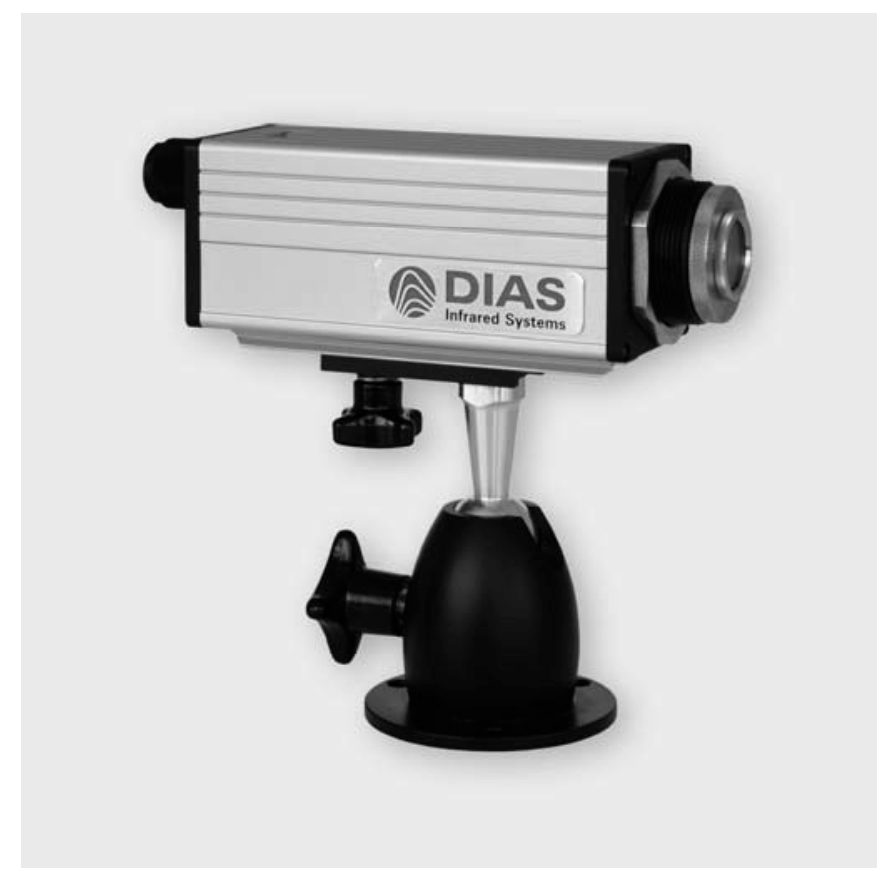

Fig.3: PYROSPOT Series 10 with focusable optics And through lens sighting

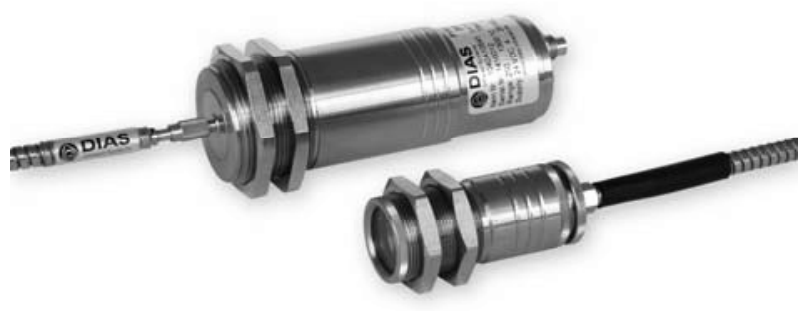

Fig.2: PYROSPOT Series $\mathbf{4 0}$ and $\mathbf{4 4}$ with fibre optics

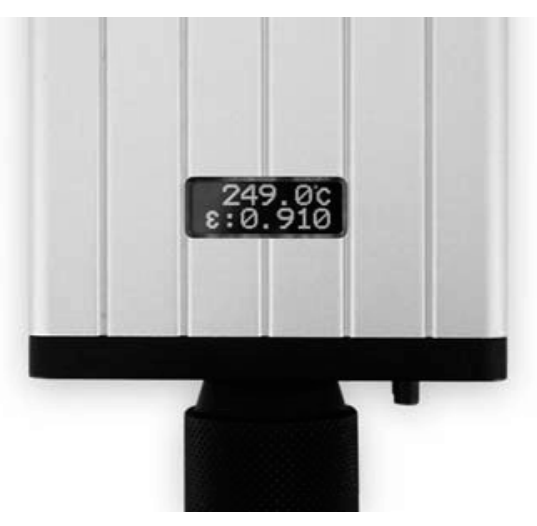

Fig.2: PYROSPOT Series 10 with display 
Table 2: Overview technical data PYROSPOT series 40 and 44

\begin{tabular}{|c|c|c|c|c|}
\hline Type & $\begin{array}{l}\text { DS } 40 \mathrm{~N} \\
\text { DS } 44 \mathrm{~N}\end{array}$ & $\begin{array}{l}\text { DSF 40N } \\
\text { DGF 44N }\end{array}$ & $\begin{array}{l}\text { DG } 40 \mathrm{~N} \\
\text { DG } 44 \mathrm{~N}\end{array}$ & $\begin{array}{l}\text { DGF 40N } \\
\text { DSF 44N }\end{array}$ \\
\hline Temperature range & \multicolumn{2}{|c|}{$250^{\circ} \mathrm{C}$ to $1800^{\circ} \mathrm{C}$} & \multicolumn{2}{|c|}{$600^{\circ} \mathrm{C}$ to $2500^{\circ} \mathrm{C}$} \\
\hline Spectral range & \multicolumn{2}{|c|}{$0.8 \mu \mathrm{m}$ to $1.1 \mu \mathrm{m}$} & \multicolumn{2}{|c|}{$1.5 \mu \mathrm{m}$ to $1.8 \mu \mathrm{m}$} \\
\hline Fibre optics & No & Yes & No & Yes \\
\hline Optics & \multicolumn{4}{|c|}{$\begin{array}{l}\text { Fixed- or vario optics, spot sizes from } 1.2 \mathrm{~mm} \\
\text { Vario optics, spot sizes from } 0.7 \mathrm{~m} \text { (for fibre optics devices) }\end{array}$} \\
\hline Measurement uncertainty & \multicolumn{4}{|c|}{$0.5 \%$ of meas. value } \\
\hline Response time (t95) & \multicolumn{4}{|c|}{ from $5 \mathrm{~ms}$} \\
\hline Emissivity & \multicolumn{4}{|c|}{0.05 to 1.00} \\
\hline Storage & \multicolumn{4}{|c|}{ Maximum value storage } \\
\hline Output & \multicolumn{4}{|c|}{$0 / 4$ to $20 \mathrm{~mA}$, tempearture linear } \\
\hline Interface & \multicolumn{4}{|c|}{$\begin{array}{l}\text { galvanically isolated USB interface } \\
\text { galvanically isolated RS } 485 \text { interface }\end{array}$} \\
\hline Aiming & \multicolumn{4}{|c|}{ Integrated LED- or Laser- aiming light } \\
\hline
\end{tabular}

Table 3: Overview technical data PYROSPOT series 40 and 44

\begin{tabular}{|c|c|c|c|}
\hline Type & $\begin{array}{l}\text { DT 40L } \\
\text { DT 44L }\end{array}$ & $\begin{array}{l}\text { DT 40G } \\
\text { DT 44G }\end{array}$ & $\begin{array}{l}\text { DT 40F } \\
\text { DT 44F }\end{array}$ \\
\hline Temperature range & $-40^{\circ} \mathrm{C}$ to $1000^{\circ} \mathrm{C}$ & $100{ }^{\circ} \mathrm{C}$ to $2500^{\circ} \mathrm{C}$ & $300{ }^{\circ} \mathrm{C}$ to $2500{ }^{\circ} \mathrm{C}$ \\
\hline Spectral range & 8 to $14 \mu \mathrm{m}$ & $5.14 \mu \mathrm{m}$ & $3.9 \mu \mathrm{m}$ \\
\hline Optics & \multicolumn{3}{|c|}{ Fixed optics, spot sizes from $2 \mathrm{~mm}$} \\
\hline Measurement uncertainty & \multicolumn{3}{|c|}{ from $0.6 \%$ of meas. value $+1 \mathrm{~K}$} \\
\hline Response time (t95) & \multicolumn{3}{|c|}{ from $10 \mathrm{~ms}$} \\
\hline Emissivity & \multicolumn{3}{|c|}{0.200 to 1.000} \\
\hline Storage & \multicolumn{3}{|c|}{ Maximum / minimum value storage } \\
\hline Output & \multicolumn{3}{|c|}{$0 / 4$ to $20 \mathrm{~mA}$, tempearture linear } \\
\hline Interface & \multicolumn{3}{|c|}{$\begin{array}{l}\text { galvanically isolated USB interface } \\
\text { galvanically isolated RS } 485 \text { interface }\end{array}$} \\
\hline Aiming & \multicolumn{3}{|c|}{ Integrated LED aiming light } \\
\hline
\end{tabular}

Table 4: Overview technical data PYROSPOT series 10

\begin{tabular}{|l|l|l|}
\hline Type & DS $10 \mathrm{~N}$ & DG 10N \\
\hline Temperature range & $200{ }^{\circ} \mathrm{C}$ to $250{ }^{\circ} \mathrm{C}$ & $600{ }^{\circ} \mathrm{C}$ to $3000{ }^{\circ} \mathrm{C}$ \\
\hline Spectral range & $0.8 \mu \mathrm{m}$ to $1.1 \mu \mathrm{m}$ & $1.5 \mu \mathrm{m}$ to $1.8 \mu \mathrm{m}$ \\
\hline Optics & Vario optics, spot sizes from $1.2 \mathrm{~mm}$ \\
\hline Measurement uncertainty & $0.5 \%$ of meas. value \\
\hline Response time (t95) & from $2 \mathrm{~ms}$ \\
\hline Emissivity & 0.050 to 1.000 \\
\hline Storage & Maximum value storage \\
\hline Output & $0 / 4$ to $20 \mathrm{~mA}$, tempearture linear \\
\hline Interface & galvanically isolated RS 485 interface \\
\hline Aiming & Integrated LED- or Laser- aiming light or through lens sighting \\
\hline
\end{tabular}




\section{Summary and future prospects}

Building a compact Pyrometer series is a continuous process of further development and enhancements.

Existing devices, features and accessories should be retained, improved and extended.

New device types with photoconductive detectors and ratio pyrometers based on silicon and InGaAs detectors are next steps to complete the series philosophy. Special pyrometers for measurements on certain materials, e.g. silicon, tungsten or aluminium, which are equipped with special filters, should be integrated in existing series. Also more comfortable operating elements like display and buttons must fit into the current design characteristics and accessories.

Very important is to retain uniform standards for digital interfaces and software evaluation. Even devices of different series in combination with portable parameterizing units should have identical interface and protocol structure.

\section{Literature}

/1/ MODBUS over Serial Line, Specification and Implementation Guide, V1.02, http://www.modbus.org /2/ MODBUS Application Protocol Specification, V1.1b, http://www.modbus.org 